\title{
Diagnóstico de um projeto de enriquecimento florestal na Comunidade do Brasileirinho, Manaus, Amazonas $^{1}$
}

\author{
André Luis WILLERDING ${ }^{2}$, Luiz Antonio de OLIVEIRA ${ }^{3}$.
}

\begin{abstract}
RESUMO
As ações do projeto consistiram no reconhecimento da comunidade, realizando-se entrevistas, seguidas de um levantamento da demanda por espécies florestais e frutíferas, aquisição, transporte e distribuição das mudas e sementes. Das 28 propriedades cadastradas, 18 tornaram-se participantes, engajando-se no projeto. Na comunidade, a fruticultura é a principal atividade e o cupuaçu a principal cultura, presente em oito propriedades participantes com 2950 indivíduos plantados. Outras culturas importantes são: manga, pupunha, açaí, coco. O projeto promoveu também a introdução e o plantio de espécies vegetais com valor de mercado. Com as atividades do projeto, diversificaram-se as espécies florestais e frutíferas existentes na comunidade. Distribuíram-se 12.455 mudas de pau-rosa, cedro, andiroba, mogno, maçaranduba, seringueira, camu-camu (caçari), araçá-boi, cubiu e outras. Como perspectiva, as ações pretendem fixar o agricultor no campo, propor alternativas econômicas, sensibilizar a comunidade com relação à preservação da floresta e exploração racional dos recursos naturais.
\end{abstract}

PALAVRAS CHAVES

Amazônia, Enriquecimento Florestal, Essências Florestais, Espécies Frutíferas.

\section{Diagnosis of a project of forest enrichment in the community of the Brasileirinbo, Manaus, Amazon ${ }^{1}$}

\begin{abstract}
The activities of this project consisted in the recognition of the community by interviews, followed by a survey of the demand for forest and fruitful species, the acquisition, transport and distribution of seedlings and seeds. Of the 28 registered properties, 18 participated in the project. In the community, fruit culture is the main activity, and cupuaçu the main fruit in eight participant properties with 2950 plants. Other cultures are: mango, pupunba, açai, coconut. The project also promoted the introduction and plantation of vegetal species with market value. Among the project activities, community forest and fruit species were diversified with 12,455 seedlings of wood-rose, cedar andiroba, mabogany, maçaranduba, seringueira, camu-camu, araçá-boi, cubiu and anothers were distributed. As the perspective, the activities intend to fix the agriculturist in the field, to consider economic alternatives, to make the community aware of the between preservation of the forest and the rational exploration of natural resources.
\end{abstract}

KEYWORDS

Amazon, Forest Enrichment, Rain Tropical Forest, Tropical Fruit

\footnotetext{
${ }^{1}$ Projeto desenvolvido com apoio do BASA S/A e INPA-PPI 2-3410

${ }^{2}$ Pesquisador Bolsista PCI. Instituto Nacional de Pesquisas da Amazônia -INPA, Coordenação de Pesquisas em Ciências Agronômicas - CPCA. Cx Postal 478, 69011-970. Manaus, AM Brasil. andrelw@inpa.gov.br

${ }^{3}$ Pesquisador Titulare Coordenador do Projeto. INPA/CPCA. luizoli@inpa.gov.br
} 


\section{INTRODUÇÃO}

A ocupação da Amazônia tem levado a um desenvolvimento social, ecológico e econômico insustentável (Uhl et al., 1998). O padrão de uso e ocupação da terra na região é caracterizado pela substituição da floresta nativa por sistemas agrícolas e/ou pastoris efêmeros (Gascon \& Moutinho, 1998). Isso leva a uma necessidade de recuperação florestal nas áreas alteradas visando uma manutenção dos sistemas florestais nativos e secundários através de uma produção florestal e agro-florestal com fins socioeconômicos ou de restauração ambiental da floresta.

Aliado a esse problema, a agricultura na Amazônia é em grande parte primária devido ao baixo poder aquisitivo dos agricultores, além dos problemas edafo-climáticos, de isolamento geográfico e de dificuldades de escoamento da produção (Oliveira et al., 1997; Souza, 2000). Em contraste, essa região apresenta-se como o segundo centro de origem e diversidade de espécies frutíferas domesticadas do mundo (Clement, 2000). Tal fato mostra a potencialidade regional com frutíferas e essências florestais para a geração de renda (Clay et al., 2000).

Os produtos naturais sempre estiveram presentes na economia da Amazônia, constituindo-se em alternativas de sobrevivência. Entretanto, o processo de exploração desses recursos nem sempre ocorreu de forma adequada (Revilla, 2001). As florestas, fonte de alimentos com cultivos de ciclo longo, são substituídas por culturas de ciclo curto no processo de colonização (Serrão, 1992). Com isso, as matas nativas diminuem com o aumento das populações humanas, com muitos imigrantes de outros locais que, desconhecendo a região, praticam sistemas de produção não adaptados às condições de clima e solos, reduzindo o período de pousio, iniciando-se um processo de degradação (Brienza et al., 1998; Metzger et al., 1998; Souza et al., 1999). Com a perda da produtividade, o agricultor desloca sua plantação, buscando novas áreas de floresta onde reinicia o ciclo de derrubada, queima e degradação, mantendo-se num ciclo de pobreza (Dubois, 1996).

A cidade de Manaus vem sofrendo um processo de crescimento urbano nas últimas décadas com a implantação do Complexo Industrial. Os desmatamentos das áreas de floresta com posterior ocupação humana têm sido interpretados como os primeiros passos para a modificação e degradação ambiental (Cleto Filho \& Walker, 2001), atingindoa tanto ecologica (perda de recursos naturais) quanto economicamente (fontes de renda). Dessa forma, ações que valorizem a floresta como fonte de renda e de recursos naturais devem ser pensadas como proposta de melhoria na qualidade de vida das populações locais (Gonçalves et al., 1992; Clay et al., 2000).

A Comunidade do Brasileirinho encontra-se no limite da cidade com as bordas da floresta, sendo uma área de pressão para o processo de desmatamento. Este projeto visa a modificação dessa realidade com a fixação do agricultor, promovendo o plantio de espécies importantes nas áreas desmatadas, preservando a mata nativa e aumentando a diversidade de produtos com potencial mercadológico.

\section{MATERIAL E MÉTODOS}

As ações do projeto iniciaram-se em outubro de $2000 \mathrm{com}$ o reconhecimento da comunidade, realizando-se reuniões com os produtores rurais. Numa segunda etapa (novembro/ 2000 a março/2001), realizou-se um cadastramento e formação de um banco de dados referente às propriedades, através da aplicação de um questionário sócio-econômico, onde a entrevista ocorreu em cada propriedade. Em seguida, estabeleceu-se um levantamento qualitativo (presença/ausência) e quantitativo (contagem) das principais espécies com potencial de mercado, bem como as demandas dos agricultores em relação às mudas de essências florestais e frutíferas que mais lhes convinham. A terceira etapa (março/2001 a junho/2001) constituiu na aquisição e distribuição das mudas, as quais foram adquiridas do próprio viveiro do INPA (Reserva ZF-2 - CPST) ou de viveiristas idôneos. Providenciou-se o transporte das mesmas até a comunidade, armazenando-as na propriedade de uma liderança local. Repassou-se aos agricultores a responsabilidade pelo plantio e distribuição das mudas entre si. Esta foi uma ação estratégica para que os agricultores se engajassem no projeto, promovendo um incentivo com respeito às mudas, às idéias do projeto e às suas propriedades. Na quarta fase (junho/2001 a janeiro/2002) implantou-se experimentos de adubação em culturas como camu-camu e cedro em algumas propriedades. A quinta fase (a partir de janeiro/2002) consistiu no acompanhamento dos plantios como forma de testar alternativas de baixo insumo para os agricultores (ainda em execução).

\section{RESULTADOS E DISCUSSÃO}

O cadastramento realizado em março de 2001 possibilitou uma análise mais apurada da comunidade. De todas as propriedades cadastradas (28), 18 tornaram-se propriedades participantes, engajando-se no projeto com o plantio de mudas e representando 64,3\% do total de propriedades (Tabela 1). As áreas das propriedades participantes representam $74,7 \%$ da área total cadastrada inicialmente. Embora nem todas as propriedades contatadas na primeira etapa tenham se engajado no projeto, a maioria das propriedades e cerca de $2 / 3$ da área abrangida atuam no projeto. Isto representa uma sensibilização da comunidade e um bom resultado. Para as áreas de mata nativa, objeto de preservação no projeto, $78,9 \%$ do total das áreas estão "protegidas", isto é, seus proprietários estão enriquecendo as áreas abertas, minimizando a pressão sobre as áreas de floresta. Para as áreas desmatadas, objeto de recuperação e enriquecimento no projeto, 66,3 \% estão inseridas no projeto, representando uma boa perspectiva aos objetivos propostos. Ainda como características sociais, o tempo médio de permanência dos produtores rurais na área é de 3,3 anos. Em relação às fontes de energia elétrica, somente oito propriedades das participantes a possuíam. Isto se deve à recente instalação de energia na comunidade (em outubro/ 


\section{ACTA AMAZONICA}

2000). Apenas oito propriedades participantes possuíam alguma fonte de água. Com a falta de energia reinante, fica difícil construir um poço ou puxar a água de um igarapé mais afastado. Com o ingresso da energia na comunidade, a tendência é que haja um melhora no fornecimento de água nas propriedades.

A seguir, a Tabela 2 apresenta a ocorrência e contagem das principais espécies com potencial de mercado presentes nas

Tabela 1 - Principais Resultados do Cadastro (março/2001).

\begin{tabular}{lll}
\hline \hline Propriedades & Cadastradas & Participantes \\
\hline Quantidade & 28 & 18 \\
Área total (ha.) & 221 & 165 \\
Área média (ha.) & 7,9 & 9,2 \\
Mata - área total (ha.) & 109 & 86 \\
Mata - área média (ha.) & 3,9 & 4,8 \\
$\begin{array}{l}\text { Capoeira - área total (ha.) } \\
\text { Capoeira - }\end{array}$ & 32 & 20 \\
$\begin{array}{l}\text { área média (ha.) } \\
\text { Desmatamento - }\end{array}$ & 1,2 & 1,1 \\
área total (ha.) & 89 & 59 \\
$\begin{array}{l}\text { Desmatamento - } \\
\text { área média (ha.) }\end{array}$ & 3,2 & 3,3 \\
$\begin{array}{l}\text { Tempo médio de } \\
\text { permanência (anos) }\end{array}$ & 2,5 & 3,3 \\
\hline \hline
\end{tabular}

propriedades. Não foram computadas as espécies de subsistência (horta familiar). Das 20 espécies listadas, catorze (70\%) são nativas para a Amazônia, distribuídas em doze famílias botânicas e seis (30\%) são exóticas incluídas em seis famílias botânicas. Nota-se que a fruticultura é a principal atividade agrícola e o cupuaçu a principal cultura, presente em oito propriedades participantes com 2950 indivíduos plantados. Este fato corrobora com o trabalho de Wandelli \& Souza (2000), que demonstraram em sistemas de plantio de pequenos agricultores no Amazonas, onde a fruticultura insere-se como principal atividade e o cupuaçu como a principal cultura. Isto demonstra o interesse pela seguridade alimentar, uma possibilidade de comercialização imediata e de renda mais rápida em relação ao plantio de espécies florestais.

A baixa freqüência (4/20) de espécies madeireiras nas propriedades (cumaru, mogno, angelim e jatobá) indica a dificuldade em torno da questão madeireira e de investimentos de retorno econômico de longo prazo.

A Tabela 3 apresenta a demanda e a oferta em relação às mudas e sementes de essências florestais (EF) e espécies frutíferas (FR) de importância econômica. Embora nem toda a oferta tenha suprido a demanda, em função de recursos financeiros e disponibilidade nos viveiros, considerou-se como satisfatório o volume de mudas e sementes distribuídas. Ao todo, foram distribuídas 6205 mudas e 6250 sementes, incluídas em treze espécies vegetais. Nota-se que há uma diversificação das espécies em relação às já existentes na comunidade. A

Tabela 2 - Ocorrência e contagem de espécies nativas e exóticas da Amazônia nas propriedades participantes (março/2001).

\begin{tabular}{llllll}
\hline \hline Cultura & Espécie & Família & Prop. & Plantas & Origem* \\
\hline Cupuaçu & Theobroma grandifolium & Sterculiaceae & 8 & 2950 & Nativa \\
Mamão & Carica papaya & Caricaceae & 3 & 1270 & Nativa \\
Açaí & Euterpe oleraceae & Arecaceae & 5 & 940 & Nativa \\
Pupunha & Bactris gasipae & Arecaceae & 7 & 160 & Nativa \\
Abacate & Persea americana & Lauraceae & 5 & 101 & Nativa \\
Araçá & Eugenia stipitata & Myrtaceae & 2 & 80 & Nativa \\
Abacaxi & Ananas comosus & Bromeliaceae & 2 & 60 & Nativa \\
Caju & Anacardium occidentale & Anacardiaceae & 2 & 60 & Nativa \\
Cumaru & Dipteryx odorata & Fabaceae & 1 & 51 & Nativa \\
Biribá & Rollinia mucosa & Anonaceae & 3 & 37 & Nativa \\
Mogno & Swietenia macrophylla & Meliaceae & 2 & 25 & Nativa \\
Angelim & Diniza excelsa & Lecythidaceae & 1 & 15 & Nativa \\
Castanheira & Bertholletia excelsa & Lecythidaceae & 1 & 15 & Nativa \\
Jatobá & Hymenea courbaril & Caesalpinaceae & 1 & 11 & Nativa \\
Coco & Cocos nucifera & Arecaceae & 5 & 817 & Exótica \\
Banana & Musa sp. & Musaceae & 2 & 390 & Exótica \\
Manga & Mangifera indica & Anacardiaceae & 8 & 285 & Exótica \\
Citros & Citrus sp. & Rutaceae & 3 & 65 & Exótica \\
Café & Coffea arabica & Rubiaceae & 2 & 15 & Exótica \\
Jambo & Syzygium malaccensis & Myrtaceae & 2 & 9 & Exótica \\
\hline \hline
\end{tabular}




\section{ACTA \\ AMAZONICA}

DIAGNÓSTICO DE UM PROJETO DE ENRIQUECIMENTO FLORESTAL

NA COMUNIDADE DO BRASILEIRINHO, MANAUS, AMAZONAS maioria das espécies distribuída é de essências florestais (69\%), havendo ainda com relação às frutíferas, a inserção do camucamu (caçari), araçá-boi e cubiu, antes inexistentes na comunidade. Todas as espécies distribuídas possuem valor de mercado, ou para a alimentação ou para outros mercados como cosmético ou farmacêutico, que apresentam maior valor agregado do produto e são importantes para a comercialização, tanto a médio prazo (até 5 anos, frutíferas) como a longo prazo (mais de 10 anos, essências florestais).

Na Tabela 4 constam os dados referentes às entrevistas, bem como às atividades do projeto. Observa-se que em média, a área das propriedades participantes é de 9,2 hectares, embora muitas possuam até quatro hectares (nove propriedades), com poucas (3 propriedades) com mais de 20 hectares. Em relação à área de mata nativa, em média, as propriedades possuem 4,8 hectares, indicando cerca de $52 \%$ da área média das propriedades. Para as áreas de capoeiras, local onde houve desmatamento com ressurgimento de uma mata secundária, em média, as propriedades possuem 1,1 hectare, indicando áreas se recuperando de desmatamentos recentes nestas propriedades. Em relação às áreas desmatadas, as propriedades participantes possuem uma média de 3,3 hectares. É sobre estas áreas desmatadas que se baseiam as atividades do projeto, com o replantio de frutíferas e essências florestais.

Na Figura 1, observa-se uma relação direta entre o aumento de áreas desmatadas com o tempo de permanência na comunidade $\left(R^{2}=0,98\right)$. Esta é uma tendência geral em propriedades que não possuem assistência técnica condizente, nem meio econômico para custear de forma adequada uma produção agrícola. Como conseqüência, novas áreas são desmatadas com o tempo. Nas Figuras 2 e 3, nota-se que os proprietários que mais se interessaram por mudas estavam entre os que adquiriram as propriedades mais recentemente, com até seis anos na comunidade. Elas adquiriram, em média, mais mudas frutíferas ou de essências florestais, contrastando com alguns antigos proprietários, cuja aquisição de mudas foi inferior. Isso demonstra um interesse das propriedades mais novas pelo replantio. O pouco interesse das outras propriedades advém dos seus plantios já estabelecidos, embora tenham também, maiores áreas desmatadas. Isso torna a ação do projeto importante, pois, com o estabelecimento desses plantios, a perspectiva é que ocorra uma quebra dessa tendência de desmatamento com o tempo de permanência. Se isso acontecer, o objetivo do projeto será alcançado.

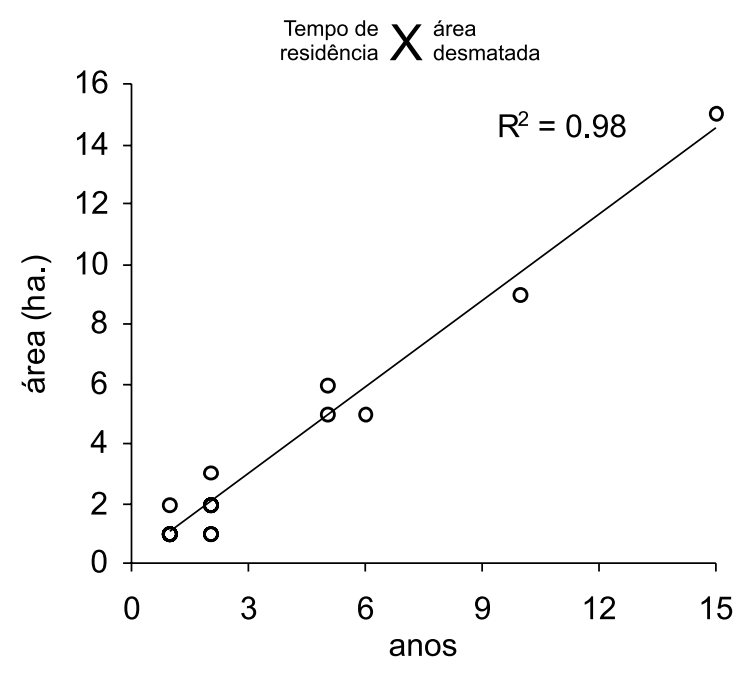

Figura 1 - Relação entre o tempo de residência x área desmatada.

Tabela 3 - Oferta e demanda de Essências Florestais (EF) e Frutíferas (FR) nativas de importância econômica.

\begin{tabular}{|c|c|c|c|c|c|c|}
\hline Tipo & Modo & Cultura & Espécie & Família & Oferta & Demanda \\
\hline $\mathrm{EF}$ & Mudas & Pau-rosa & Aniba duckei & Lauraceae & 1670 & 1150 \\
\hline FR & Mudas & Camu-camu & Myrciaria dubia & Myrtaceae & 1611 & 1800 \\
\hline EF & Mudas & Cedro & Cedrela odorata & Meliaceae & 900 & 450 \\
\hline FR & Mudas & Araçá - boi & Eugenia stipitata & Myrtaceae & 700 & 300 \\
\hline EF & Mudas & Andiroba & Carapa guianensis & Meliaceae & 300 & 250 \\
\hline EF & Mudas & Cumaru & Dipteryx odorata & Fabaceae & 300 & 400 \\
\hline EF & Mudas & Mogno & Swietenia macrophylla & Meliaceae & 300 & 400 \\
\hline EF & Mudas & Maçaranduba & Manilkara huberi & Sapotaceae & 134 & 0 \\
\hline EF & Mudas & Seringueira & Hevea brasiliensis & Euphorbiaceae & 132 & 110 \\
\hline EF & Mudas & Cupuaçu & Theobroma grandifolium & Sterculiaceae & 108 & 100 \\
\hline EF & Mudas & Jatobá & Hymenea courbaril & Caesalpinaceae & 50 & 20 \\
\hline FR & Mudas & Araçá - pêra & Psidium acutangulum & Myrtaceae & 0 & 300 \\
\hline \multirow[t]{2}{*}{ FR } & Sementes & Cubiu & Solanum sessiliflorum & Solanaceae & 6250 & 2000 \\
\hline & & & & Total & 12455 & 7280 \\
\hline
\end{tabular}


Tabela 4 - Relação entre tempo, áreas de mata, capoeira e desmatada e espécies adquiridas do projeto pelas propriedades participantes.

\begin{tabular}{|c|c|c|c|c|c|c|c|}
\hline \multirow{2}{*}{$\begin{array}{l}\text { Propriedades } \\
\text { Participantes }\end{array}$} & \multirow{2}{*}{$\begin{array}{l}\text { Tempo de } \\
\text { Residência (anos) }\end{array}$} & \multicolumn{4}{|c|}{ Área (hectares) } & \multicolumn{2}{|c|}{ Espécies } \\
\hline & & Total & Mata & Capoeira & Desmatada & Frutíferas (FR) & Florestais (EF) \\
\hline 1 & 2 & 4 & 1 & 1 & 2 & 1279 & 500 \\
\hline 2 & 1 & 4 & 2 & 1 & 1 & 50 & 50 \\
\hline 3 & 1 & 4 & 2 & 1 & 1 & 1250 & 300 \\
\hline 4 & 10 & 11 & 1 & 1 & 9 & 300 & 150 \\
\hline 5 & 1 & 4 & 2 & 1 & 1 & 50 & 0 \\
\hline 6 & 1 & 4 & 2 & 1 & 1 & 0 & 50 \\
\hline 7 & 15 & 31 & 15 & 1 & 15 & 540 & 50 \\
\hline 8 & 1 & 4 & 2 & 1 & 1 & 0 & 250 \\
\hline 9 & 6 & 29 & 23 & 1 & 5 & 360 & 50 \\
\hline 10 & 2 & 7 & 3 & 2 & 2 & 600 & 300 \\
\hline 11 & 5 & 9 & 2 & 1 & 6 & 1264 & 680 \\
\hline 12 & 1 & 4 & 2 & 1 & 1 & 0 & 100 \\
\hline 13 & 5 & 9 & 2 & 2 & 5 & 2140 & 420 \\
\hline 14 & 1 & 22 & 19 & 1 & 2 & 697 & 300 \\
\hline 15 & 2 & 6 & 2 & 1 & 3 & 0 & 150 \\
\hline 16 & 2 & 5 & 2 & 1 & 2 & 0 & 150 \\
\hline 17 & 2 & 4 & 2 & 1 & 1 & 0 & 100 \\
\hline 18 & 2 & 4 & 2 & 1 & 1 & 0 & 325 \\
\hline Médias & 3,3 & 9,2 & 4,8 & 1,1 & 3,3 & 474 & 218 \\
\hline
\end{tabular}

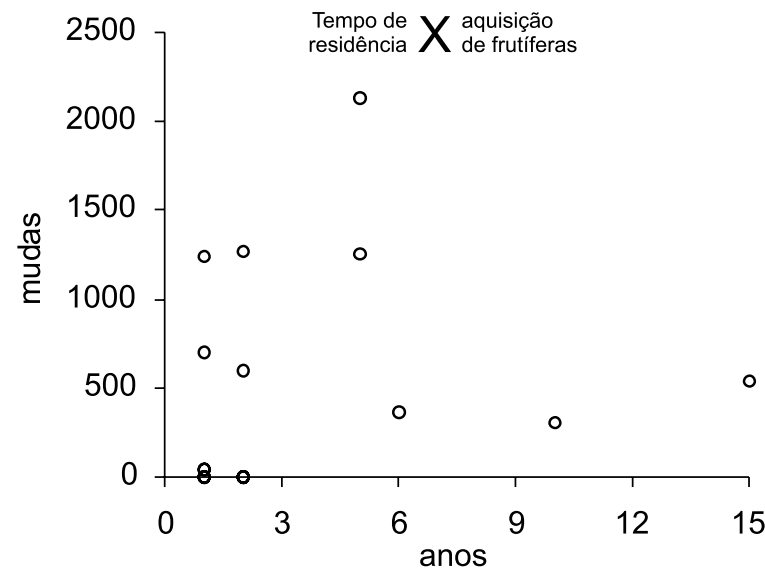

Figura 2 - Relação entre o tempo de residência e o total de mudas de frutíferas adquiridas pelos produtores.

\section{CONCLUSÃO}

A participação da comunidade aos objetivos propostos está demonstrada pelo interesse pelo plantio de espécies frutíferas e de essências florestais.O projeto sensibilizou a maioria dos proprietários rurais da comunidade do Brasileirinho, onde $64 \%$ das propriedades se envolveram com as atividades do projeto,

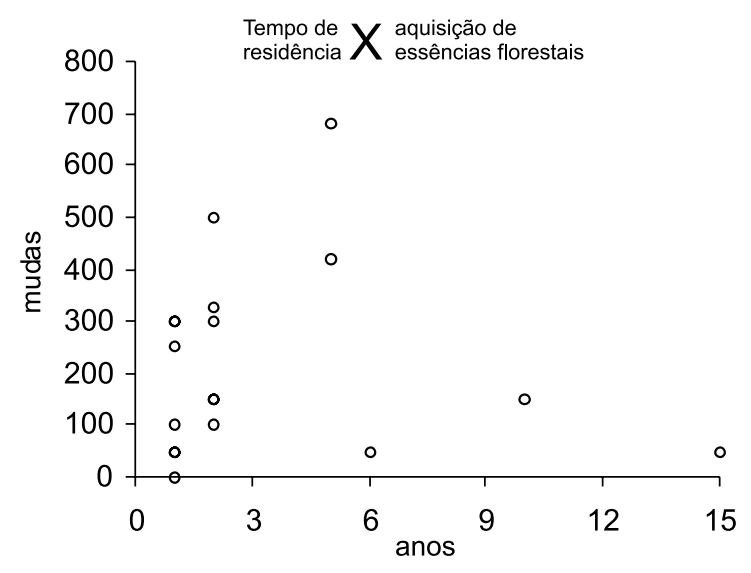

Figura 3 - Relação entre o tempo de residência e o total de mudas de essências florestais adquiridas pelos produtores.

adquirindo mudas e promovendo um enriquecimento do seu sistema produtivo.

A maioria das espécies distribuída é de essências florestais (69\%), havendo ainda com relação às frutíferas, a inserção do camu-camu, araçá-boi e cubiu, antes inexistentes na comunidade. 


\section{BIBLIOGRAFIA CITADA}

Brienza, J.R.; Santos, W.E.; Costa, V.D.; Pantoja, R.F.R.; Vielhauer, K.; Denic, M; Vleb, P.G.L. 1998. Changing the slash-andburn agriculture in Brazilian Eastern Amazonia by enriching the fallow vegetation. In: Proceedings of the Third ShiftWorkshop. Manaus, Amazonas. p.109-112.

Clay, J.W.; Sampaio, P.T.B; Clement, C. 2000. Biodiversidade amazônica: exemplos e estratégias de utilização. $1^{\mathrm{a}}$ Edição. Manaus. Amazonas. Programa de Desenvolvimento Empresarial e Tecnológico. SEBRAE/INPA. 409p.

Clement. C. R. 2000. Domestication of Amazonian fruit-crop: past, present and future. In: Vieira, I. C. C.; Silva, J. M. C.; Oren, D. C.; Dincão, M. A. (Eds). Diversidade Biológica e Cultural da Amazônia. Museu Paraense Emílio Goeldi. Belém, PA, 347-367p.

Clement, C. R.; Noda, H.; Noda, S. N.; Martins, A. L. U.; Silva, Gleissimar, C. 2001. Recursos Frutícolas na Várzea e na Terra Firme em Onze Comunidades Rurais do Alto Solimões, Amazonas, Brasil. Acta Amazonica 31(3): 521-527.

Cleto Filho, S. E. N; Walker, I. 2001. Efeitos da ocupação urbana sobre a macrofauna de invertebrados aquáticos de um igarapé da cidade de Manaus/AM - Amazônia Central. Acta Amazonica 31(1):69-89.

Dubois, J. C. L. 1996. Manual Agroflorestal para a Amazônia. Volume I. Rio de Janeiro. Brasil. REBRAF. 28p.

Gascon, C.; Moutinho, P. 1998. Floresta Amazônica: Dinâmica, Regeneração e Manejo. INPA/MCT Manaus, Amazona. 373p.

Gonçalves, J.L. de M.; Kageyama, P.Y.; Freixêdas, F.; Gonçalves, J.C.; Geres, W.L. de A. 1992. Capacidade de absorção e eficiência nutricional de algumas espécies arbóreas tropicais. In: Anais do Congresso Nacional Sobre Essências Nativas, 2, São Paulo. São Paulo. p.463-469.

Metzger, J.P.; Denich, M. Vielhauer, K. 1998. Fallow periods and landscape structure in areas of slash-and-burn agriculture (NE Braziliam Amazon). In: Proceedings of the Third ShiftWorkshop. Manaus, Amazonas. p.95-99.

Oliveira, L.A; Moreira, F.W.; Moreira, F.M.S. 1997. Ocorrência de microrganismos benéficos em ecossistemas amazônicos. In: Noda, H.; Souza, L.A.G.; Fonseca, O.J.M. (eds.) Duas Décadas de Contribuição do INPA à Pesquisa Agronômica no Trópico Úmido. (1997), p. 221-240.
Revilla, J. 2001. Plantas da Amazônia: oportunidades econômicas e sustentáveis. SEBRAE/INPA. Manaus, Amazonas. 405p.

Serrão, E. A. S. 1992. Possibilities for sustainable agricultural and forestry development in the Brazilian Amazon: an EMBRAPA proposal. In: Conference on Environmentally sound socio Economic in the development in the Humid Tropics. Manaus, Brasil. p.29-32.

Souza, G.F.; Guimarães, R.R..; Souza, N.R.; Nunes, J.S.; Lourenço, J.N.P. 1999. Multi-strata agroforestry as an alternative for small migrant farmers practicing shifting cultivation in Central Amazonian communities in Brazil. In: Jiménez, F.; Beer, J. (Compilors). International Symposium "MultiStrata Agroforestry Systems with Perennial Crops. Turrialba, Costa Rica. p.243-246.

Souza, G. F. 2000. Manejo do solo e seu efeito no crescimento e produção do cupuaçuzeiro (Theobroma grandiflorum (Willdenow ex Spreng) Schumann), na composição florística e biomassa de plantas invasoras em sistemas agroflorestais no município de Presidente Figueiredo, Amazonas. Tese de Doutorado. Manaus Instituto Nacional de Pesquisas da Amazônia/Universidade Federal do Amazonas. Manaus, AM. $161 \mathrm{p}$.

Uhl, C.; Barreto, P.; Veríssimo, A.; Barros, A. C.; Amaral, P.; Vidal, E. 1998. Uma abordagem integrada de pesquisa sobre o manejo dos recursos florestais na Amazônia brasileira. In: Gascon, C.; Moutinho, P. (Eds.). Floresta Amazônica: Dinâmica, Regeneração e Manejo. Instituto Nacional de Pesquisas da Amazônia/Ministério de Ciência e Tecnologia. Manaus, p.313-331.

Wandelli, E. V.; Souza, M. P. S. 2000. Análise da sustentabilidade de sistemas agroflorestais do Estado do Amazonas através de sua diversidade florística. In: III Congresso Brasileiro de Sistemas Agroflorestais. Manaus. Livro de Resumos Expandidos, p26-28.

\section{RECEBIDO EM 01/08/2003 ACEITO EM 12/09/2005}

